\title{
Proceso de admisión y curso de nivelación en el ingreso a la universidad. Un estudio de caso
}

\section{Admission process and leveling course in the entrance to the university. A case study}

Fabián Eugenio Bravo Guerrero

Universidad de Cuenca, Ecuador

Lourdes Illescas-Peña

Universidad de Cuenca, Ecuador

Tatiana Quezada

Universidad de Cuenca, Ecuador

Autor para correspondencia: fabianbravo@yahoo.com, lourdes.illecasp@ucuenca.edu.ec, tatiana.quezada@ucuenca.edu.ec

Fecha de recepción: 23 de abril de 2018 - Fecha de aceptación: 23 septiembre de 2018

Resumen: Existen críticas al sistema de ingreso a la universidad ecuatoriana debido a que muchos estudiantes no acceden a las carreras de su preferencia o vocación; por eso, se realizó una investigación al proceso de admisión y curso de nivelación que tomaron los estudiantes que ingresaron a la Facultad de Filosofía, Letras y Ciencias de la Educación de la Universidad de Cuenca. Es importante evidenciar con cifras la realidad de estas afirmaciones, para esto se estudió a los estudiantes que fueron admitidos e ingresaron al curso de nivelación entre septiembre del 2015 a febrero del 2016, mediante encuestas al inicio y final del curso. En base a los resultados de las notas del Examen Nacional para la Educación Superior -ENES- con que ingresaron estudiantes a las carreras, se comparó con las carreras de preferencia, y la experiencia luego de tomar el curso de nivelación en la facultad.

Palabras clave: admisión, curso de nivelación; examen ENES; ingreso a universidad

\begin{abstract}
There are some criticisms of the access system to the ecuadorian university because many students fail to achieve access to the careers of their preference or vocation; for that reason, an investigation was made to the admission process and leveling course taken by the students who entered the Faculty of Philosophy, Letters and Education Sciences of the University of Cuenca. It is important to demostrate with real values these statements. To achieve this, students were analyzed who were admitted and entered the leveling course between September 2015 and February 2016, through surveys at the beginning and end of the course. Based on the results of the scores of the National Exam for Higher Education -ENES- with which the students entered the careers, it was compared with the careers of preference, and the experience after taking the leveling course in the faculty.
\end{abstract}

Key Words: admission; ENES exam; leveling course; admission to university 


\section{Introducción}

El período de transición entre el colegio y la universidad trae dificultades a los estudiantes, que deben afrontar una decisión importante por la carrera que desean cursar, obtener un cupo en la universidad, y una vez que ha conseguido ingresar, adaptarse al nuevo ambiente universitario. Para esto la Ley de Educación Superior, LOES, dispuso que el ingreso a la universidad ecuatoriana se dé mediante un proceso de admisión que asegure iguales oportunidades a todos los aspirantes, y que se base en los méritos, estos se valoraban en una prueba denominada Examen Nacional para la Educación Superior, ENES, si se obtenía el suficiente puntaje se conseguía un cupo en una de las carreras de preferencia del aspirante. Posterior al proceso de ingreso, se realizó un curso de nivelación de un semestre de duración con el propósito de nivelar los diversos niveles de conocimientos que traían los estudiantes.

En la Facultad de Filosofía, Letras y Ciencias de la Educación se llevó a cabo todo este proceso que se describe en detalle a continuación, además, se recopiló importante información de este proceso en cada una de las carreras.

\section{Marco Teórico}

El ingreso a la educación superior es una delicada etapa de transición para el estudiante, donde la universidad ecuatoriana debe ajustar sus acciones para que este sea un evento natural (Buitrón, Ortiz \& Yupangui, 2017), en esta etapa el estudiante atraviesa una serie de dificultades, que van desde la falta de bases disciplinares (Himmel, 2002) que traen de su formación en el colegio, a la poco acertada elección de la carrera universitaria (Valladão, Pomeroy \& Salgado, 2013) debido a que no se informan lo suficiente (Ennis \& Porto, 2001), la angustia e incertidumbre en el proceso (Viera, 2017). Una vez en la universidad los estudiantes tienen problemas de integración académica y social (Cantarero,2016) que se traducen en problemas de baja autoestima, con la consecuente baja de expectativa de éxito (Ornelas Contreras et al., 2013), hasta altos niveles de exigencia académica en la nueva carrera (Vries, Romero \& Hernández, 2011), evaluación basada en la memorización (Bravo, Trelles \& Barrazueta, 2017), docentes con un carácter excesivamente disciplinario de la formación donde no se construye el saber (Romo, Córdova \& Cueva, 2014). Existen también aspectos personales como la falta de recursos económicos (Vélez \& López, 2004) y diversos problemas familiares (López-Justicia et al., 2008) que dificultan la integración del nuevo estudiante a la vida universitaria.

Dado que estos problemas mencionados requerían ser minimizados, autoridades del gobierno, basadas en la Constitución del Ecuador (1998) y en el Plan nacional de Buen Vivir 20132017, aplicaron en las instituciones de educación superior públicas, un proceso unificado de admisión a las universidades, y un período de nivelación de un semestre, con el propósito de elevar el nivel académico del estudiante. Este proceso se implementa debido a que las universidades "deben ser incluyentes y no marginar a los usuarios (estudiantes, profesionales) por diferencias en el estatus económico, etnia, acceso geográfico, idioma, tipo de colegio de procedencia, ideología" (Romero, Bermeo \& Ruiz, 2014, p. 30), se buscaba garantizar a los ciudadanos el acceso universal a la educación pública y gratuita (Constitución, 1998, Art 28), logrando aumentar de la tasa de asistencia a la educación superior -para personas de 18 a 24 años-, de 13,54\% en el año 2001 a 22,6\% en el 2010 (Plan nacional del Buen Vivir 2013-2017, p. 164), esta política pretendía que todos los ciudadanos puedan tener igualdad de oportunidades, y su acceso a la formación académica y profesional esté en función de los méritos (LOES, 2010, Art. 4), también, la gratuidad 
ha sido un factor que ha ayudado a eliminar barreras de tipo económico, incrementar la matrícula y democratizar el acceso (Ramírez, 2013).

La Secretaría Nacional de Educación Superior, Ciencia, Tecnología e Innovación SENESCYT (LOES, 2010, Art. 183), reglamentó el Sistema Nacional de Nivelación y Admisión SNNA (2013), como consta en la LOES, 2010, Art. 81, que regulaba el sistema para el ingreso a la educación superior ecuatoriana (Constitución, 1998, Art. 356) mediante un proceso de admisión a una carrera, y la posterior nivelación de sus conocimientos para afrontar los estudios universitarios.

El proceso de admisión buscaba asegurar la igualdad de oportunidades para todos los aspirantes que ingresan a la universidad, mediante un sistema que valore los méritos del estudiante (LOES, 2010, Art. 4); mientras, la nivelación buscaba articular la educación de bachillerato con la educación superior (Constitución, 1998, Art 344), tratando de equiparar el nivel de conocimientos que los estudiantes traían desde el bachillerato general unificado -de reciente implementación en la educación secundaria-, para que el ingreso a la universidad no plantee grandes dificultades en el aspecto académico.

En primera instancia, el estudiante rendía el Examen Nacional para la Educación Superior ENES, un examen que evalúa aptitudes y consta de tres partes: razonamiento abstracto, verbal y numérico. Una vez rendido el examen ENES, recibe la calificación sobre un máximo de 1000 puntos. Era un examen con un formato diferente al que está acostumbrado el estudiante, por lo que tenía que familiarizarse con su estructura, para esto algunos colegios preparaban a sus estudiantes con este nuevo formato; pero en general, los aspirantes debían aprender a resolver tareas de razonamiento que dependían de apoyo "extracurricular" cuyo costo recaía sobre las familias de los estudiantes (Zambrano, 2016), en ese sentido Cabrera (2017) indica que, en la admisión a las universidades públicas se evidencia un enfoque que segrega al aspirante antes que ser un igualitario, y los cursos privados de formación para la prueba ENES no son para las personas de un nivel socioeconómico poco favorecido, "lejos de ser detonante de un proceso de democratización, legitimó y cubrió las desigualdades estructurales previas que diferenciaban a unos y otros postulantes" (Ospina, Moreno, Cielo \& Cabrera, 2017, p. 253).

Una vez que el aspirante conocía su nota ENES, podía postular en orden de preferencia a cinco carreras, en el caso de las carreras de educación era necesario tener un puntaje de al menos 800/1000. El SNNA, a través de un sistema informático automatizado, asignaba los cupos en función de tres variables: la nota ENES obtenida, el orden de preferencia registrado y la cantidad de cupos ofertados por la universidad. En esa asignación resultaban muchos estudiantes enviados a carreras que, aunque las escogieron en la postulación, no era su primera opción, esta asignación ocasionó que algunos terminen desertando del sistema. (Bravo, Illescas, Larriva \& Peña, 2017).

El curso de nivelación tenía una duración de un semestre, la SENESCYT organizaba los cursos, el plan curricular, los listados de estudiantes, y se llevaban a cabo en las propias Instituciones de Educación Superior -IES-. Se impartieron cinco asignaturas, algunas de ellas pudieron ser modificadas por las carreras para ajustar a sus requerimientos. Esto ocasionó que algunas carreras decidan incorporar contenidos útiles para la formación del profesional, pero que 
no ayudan a equiparar los conocimientos que traían desde el bachillerato (Bravo, et al., 2017), fin principal de la creación del curso de nivelación.

El semestre de nivelación tuvo un complejo sistema de evaluación, que requirió que el estudiante haya aprobado cada materia con un mínimo de 6,5/10, pero haber obtenido al menos 8/10 como promedio de todas las materias, además, tener al menos el $75 \%$ de asistencia; requerimientos muy superiores al que exigen las carreras de la Universidad de Cuenca con mínimos de $65 \%$ de asistencia y $60 \%$ en aprovechamiento.

En la Facultad de Filosofía, Letras y Ciencias de la Educación el estudiante podía optar por diez carreras diferentes, siete de ellas de educación que requerían de una nota ENES de al menos 800 puntos: Filosofía, Sociología y Economía, Historia y Geografía, Lengua, Literatura y Lenguajes Audiovisuales, Lengua y Literatura Inglesa, Matemáticas y Física, Cultura Física, Educación General Básica; y, tres carreras que requerían al menos 700 puntos: Ciencias de la Comunicación Social en Periodismo y Comunicación Digital, Ciencias de la Comunicación Social en Comunicación Organizacional y Relaciones Públicas, Cine y Audiovisuales.

\section{Metodología}

La investigación es un estudio de caso de la Facultad de Filosofía, Letras y Ciencias de la Educación, de la Universidad de Cuenca, se realizó un estudio transversal puramente descriptivo, que caracterizó el proceso de admisión a la universidad y el curso de nivelación de conocimientos previo al ingreso a las carreras que ofertaba la facultad. La investigación recopila información de los 403 estudiantes que ingresaron al ciclo de nivelación en septiembre del 2015 hasta febrero del 2016 a la facultad, mediante una encuesta estructurada que recoge datos del proceso de admisión a las carreras de la facultad, se recopilan datos de sus estudios secundarios, datos de su proceso de admisión a la facultad, véase la tabla 1; y, otra encuesta estructurada al finalizar el curso de nivelación a los estudiantes que asisten a clases en febrero del 2016, en esta ocasión se recopilan datos acerca de su experiencia en las diferentes asignaturas del curso de nivelación organizado por la SENESCYT.

\section{Tabla 1}

Estudiantes que ingresan al curso de nivelación en septiembre del 2015 en la Facultad de Filosofía, Letras y Ciencias de la Educación de la Universidad de Cuenca

\begin{tabular}{|c|c|}
\hline \multicolumn{2}{|c|}{ Resultados del Proceso de Admisión y Nivelació } \\
\hline Carrera & Ingresan \\
\hline Lengua y Literatura & 32 \\
\hline Historia y Geografía & 33 \\
\hline Filosofía Sociología y Economía & 35 \\
\hline Matemáticas y Fisica & 46 \\
\hline Educación General Básica & 59 \\
\hline Comunicación Social & 61 \\
\hline Cultura Física & 68 \\
\hline Lengua Inglesa & 69 \\
\hline Total & 403 \\
\hline
\end{tabular}

Los estudiantes que ingresaron a nivelación de la Facultad de Filosofía, Letras y Ciencias de la Educación de la Universidad de Cuenca tuvieron en la prueba ENES un promedio de 798 
puntos, con un máximo de 960 y un mínimo de 656 puntos como se observa en la Figura 1. En las carreras de educación se requería de 800 puntos para el ingreso, aquellos estudiantes que no obtuvieron ese puntaje ingresaron condicionados, y semanas después, tuvieron la oportunidad de rendir una nueva prueba ENES que les daba la oportunidad obtener al menos esos 800 puntos, en caso de no llegar a ese mínimo no se podía ingresar a una carrera del área de educación.

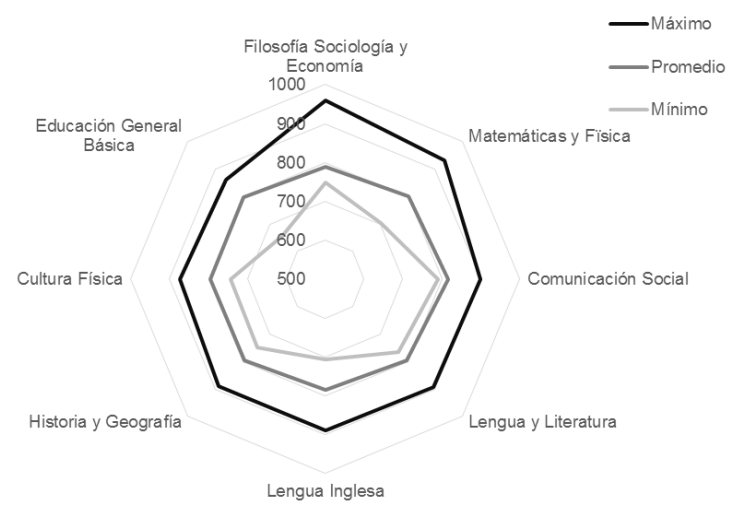

Figura 1. Notas del examen ENES de los estudiantes que ingresaron a las carreras de la Facultad de Filosofía, Letras y Ciencias de la Educación.

Los estudiantes, en su mayoría fueron recién graduados: casi la mitad de los estudiantes un 48,5\%- se había graduado hace un par de meses, un 82,8\% en los últimos dos años, y un $95,9 \%$ en los últimos cinco años.

El tipo de colegio del que proceden es un $62 \%$ de Bachillerato general unificado que no tiene ningún tipo de especialización, un 32\% vienen de un colegió técnico, un $1 \%$ de colegios con bachillerato internacional.

Se les consultó si la carrera asignada era su primera opción escogida al postular, en promedio un 52,5\% dijo no; hay que destacar que a la carrera de Filosofía solo un $14 \%$ lo escogió como primera opción. En la carrera de Matemáticas y Física, la mayoría -un 74\%prefería una carrera técnica como Arquitectura o Ingeniería. En la carrera de Lengua, Literatura y Lenguajes Audiovisuales, un 69\% la escogió como su primera opción, véase la figura 2.

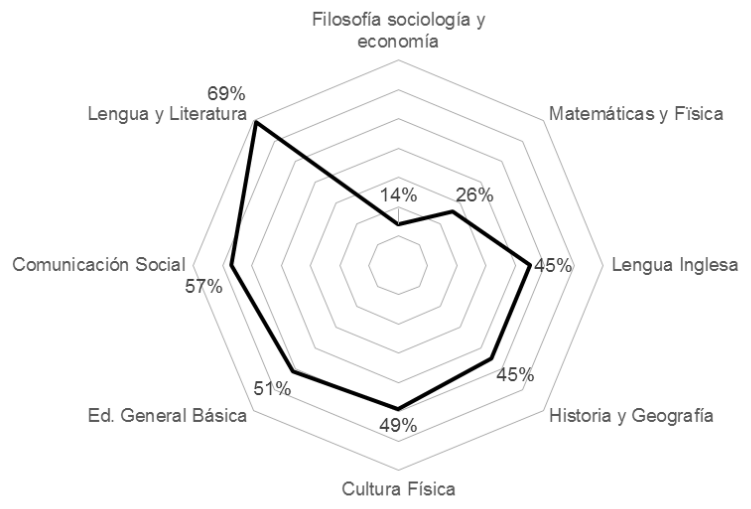

Figura 2. ¿La carrera que cursa, fue su primera opción al postular? 
Un 47,5\% escogió como primera opción las carreras de la Facultad de Filosofía, Letras y Ciencias de la Educación; mientras, el resto escogió una gran diversidad de carreras de la Universidad de Cuenca, un 14,4\% carreras técnicas, un 9,4\% escogió psicología, un 6,4\% carreras del área de la salud, un 5,9\% carreras del área de economía, y un 16,4\% en otras carreras.

Al finalizar la nivelación, se realizó una segunda encuesta donde los estudiantes dieron sus opiniones acerca de los docentes del curso de nivelación, indicaron que en las clases se utilizan diversas estrategias, como las de trabajo grupal, de investigación, estudio de casos, visitas de campo entre otras. Los principales recursos que utilizaron los docentes del curso de nivelación son la pizarra, el proyector, material concreto, videos, en menor medida usan software y simuladores. Con respecto a las evaluaciones, ellos indicaron que tienen algunas dificultades: que el tiempo es muy corto, que se ponen muy nerviosos, que se acumulan demasiados temas, que las preguntas son confusas o ambiguas, problemas de elevada complejidad, en algunos casos las preguntas son de tipo memorístico, entre otras razones. Un $82 \%$ de los estudiantes consideran que los docentes de nivelación tienen un alto dominio de la disciplina que imparten.

El curso de nivelación tenía cinco asignaturas: Sociedad y Cultura, Universidad y Buen Vivir -UBV-, Introducción al Conocimiento, Psicología y Matemáticas. Dado que los estudiantes ingresan a un programa de nivelación para una facultad del área social, un 64\% encontró las mayores dificultades en la asignatura Matemáticas. Hay que destacar que el currículo de Matemáticas, solamente consideraba reforzar los conocimientos que habían desarrollado en el bachillerato y no incluir temas nuevos.

\section{Conclusiones}

Los estudiantes que ingresaron a las carreras de la Facultad tuvieron un promedio del examen ENES de 798 puntos, en gran medida se debe a la exigencia de obtener al menos 800 puntos para ingresar a las carreras de educación, y aquellos que no obtuvieron ese puntaje tuvieron otra oportunidad de rendir el examen y obtener el mínimo requerido, fue positiva la exigencia para las carreras de educación, ya que ingresaron estudiantes con una alta nota ENES y han tenido un buen desempeño. El proceso de admisión aplicado por el SNNA mediante el sistema informático automatizado, asignó un 52,5\% de estudiantes a carreras que ellos no tenían como su primera opción, una de las carreras tenía tan solo un $14 \%$ de estudiantes que la escogieron como su primera opción, esto sucede porque hay la aspiración de los estudiantes a ingresar a las carreras tradicionales, y esos cupos se saturan muy pronto y con los puntajes más altos, quedándole al sistema informático asignar las siguientes opciones elegidas por el aspirante.

El curso de nivelación previo al ingreso a la Facultad organizado por la SENESCYT tuvo una duración de un semestre, con un complejo sistema de evaluación que fue mucho más exigente (8/10), que lo requerido en la posterior carrera universitaria (6/10). Estas reglas, propias del curso de nivelación dificultaron al estudiante que recién ingresó a adaptarse al ambiente social y académico de la universidad. El curso de nivelación incluyó algunas asignaturas que los estudiantes no consideran relevantes para su formación, también, consideran que las evaluaciones fueron complejas, privilegiaban la memoria, y muchos problemas planteados fueron confusos y rebuscados. Los docentes usaron la tradicional pizarra y el proyector para diapositivas y videos, como principales recursos. Es evidente que los docentes de nivelación 
hicieron esfuerzos por usar estrategias metodológicas y recursos didácticos que dan importancia al aprendizaje del estudiante, para pasar "de una instrucción transmisora de conocimientos a un aprendizaje para el desarrollo personal” (Ruiz, Torres \& García, 2017, p.11).

Este modelo de postulación y admisión a las carreras fue modificado para el proceso de ingreso en septiembre del 2016, mientras el curso de nivelación organizado por la SENESCYT fue aplicado hasta febrero del 2016, para la siguiente convocatoria, en septiembre del 2016 el curso de nivelación fue propuesto y organizado por cada institución educativa de educación superior.

\section{Bibliografía}

Bravo, F., Illescas, L., Larriva, S. \& Peña, M. (2017). Causas de la deserción en el ingreso a la universidad. Un estudio de caso. Revista de la Facultad de Ciencias Químicas de la Universidad de Cuenca. 18, 48-59.

Bravo, F., Trelles, C. \& Barrazueta, J. (2017). Reflexiones sobre la evolución de la clase de matemáticas en el bachillerato ecuatoriano. INNOVA Research Journal, 2(7), 1-12.

Buitrón, L., Ortiz, J. \& Yupangui, Y. (2017). Nivelación propuesta por la SENESCYT: Vivencias en la Universidad Central del Ecuador. INNOVA Research Journal, 2(10), 42-53.

Cabrera, S. (2017). The Oppressive Nature of Admission Tests to Public Higher Education. INNOVA Research Journal, 2(6), 94-99.

Cantarero, P. M. P. (2016). Factores relacionados con la deserción en el primer y segundo año de estudio en la carrera de Enseñanza de la Matemática de la Universidad Nacional de Costa Rica. Revista Electrónica Educare, 20(1).

Constituyente, Asamblea. (1998). Constitución de la República del Ecuador. Quito.

Ennis, H. M., \& Porto, A. (2001). Igualdad de Oportunidades e Ingreso a la Universidad Pública en la Argentina. Documentos de Trabajo.

Himmel, E. (2002). Modelos de análisis de la deserción estudiantil en la educación superior. Revista calidad de la educación, 17, 91-108.

López-Justicia, M., Hernández, C. M., Fernández Jiménez, C., Polo Sánchez, T., \& Chacón López, H. (2008). Características formativas y socioafectivas del alumnado de nuevo ingreso en la Universidad. Electronic Journal of Research in Educational Psychology, 6(14).

Nacional, Asamblea. (2010). Ley orgánica de educación superior, Quito.

Ornelas Contreras, M., Blanco Vega, H., Gastélum Cuadras, G., \& Muñoz Beltrán, F. (2013). Perfiles de autoeficacia en conductas académicas en alumnos de primer ingreso a la universidad. Revista electrónica de investigación educativa, 15(1), 17-28. 
Ospina, P., Moreno, K., Cielo, C., \& Cabrera, S. (2017). Las reformas universitarias en Ecuador (2009-2016): extravíos, ilusiones y realidades.

Ramírez, R. (2013) Tercera ola de transformación de la educación de la educación superior en el Ecuador. SENESCYT.

Romero, A., Bermeo, M., \& Ruiz, E. (2014). La institución de educación superior camino a la excelencia. Análisis de la aplicación del modelo de calidad en la universidad. Deserción, calidad y reforma universitaria. Apuntes para el debate, 14-33.

Romo, M. P., Córdova, P., \& Cueva, L. (2014). Debates y desafíos en la enseñanza del derecho en Ecuador. Deserción, calidad y reforma universitaria. Apuntes para el debate, 66-87.

Ruiz, L., Torres, G., \& García, D. (2017). Desafíos de la Educación Superior. Consideraciones sobre el Ecuador. INNOVA Research Journal, 3(2), 8-16.

SENESCYT. (2013). Reglamento del sistema nacional de nivelación y admisión SNNA. Quito.

SENPLADES. (2013). Plan Nacional del Buen Vivir 2013-2017. Quito.

Valladão, M. Pomero y, D. \& Salgado, A. (2013). "Estudio de un caso: La deserción de estudiantes de primer año en la Escuela de Química de la Universidad Federal de Río de Janeiro (UFRJ)". Avances en Ciencias e Ingeniería, 4(3), 63-72.

Vélez, A. López, D. (2004). Estrategias para vencer la deserción universitaria, Educación y educadores, 7, 177-203.

Viera, P. (2017). Los que "triunfan" y los que "fracasan" (Des) igualdades sociales, logros educativos y emociones: el ingreso a la universidad pública en el Ecuador de las oportunidades. Conferencia Nacional "Educación Superior en el Ecuador. Investigaciones para una Nueva Agenda de Cambios Necesarios” (págs. 1-29). Quito: UASB-Digital.

Vries, W. D. León, P. Romero, J. \& Hernández, I. (2011). ¿Desertores o decepcionados? Distintas causas para abandonar los estudios universitarios, Revista de la educación superior, 40(160), 29-49.

Zambrano-Ramírez, J. (2016). Una mirada crítica al examen nacional para la educación superior en Ecuador. Revista EDUSOL, 16(56), 37-51. 